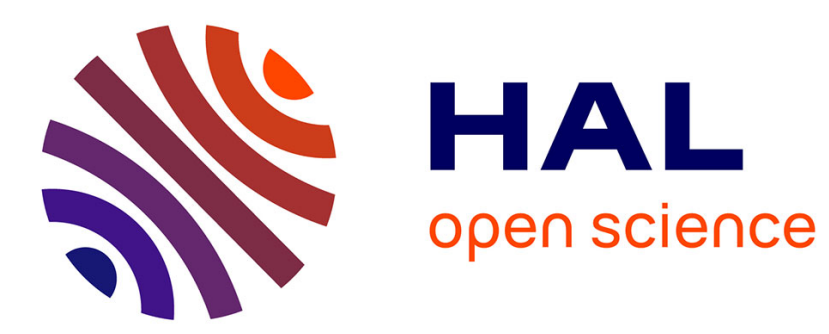

\title{
Limite de la région quasi neutre dans un semiconducteur
}

\author{
Z.T. Kuznicki, B. Zimmermann
}

\section{To cite this version:}

Z.T. Kuznicki, B. Zimmermann. Limite de la région quasi neutre dans un semiconducteur. Revue de Physique Appliquée, 1989, 24 (9), pp.851-860. 10.1051/rphysap:01989002409085100 . jpa-00246108

\section{HAL Id: jpa-00246108 https://hal.science/jpa-00246108}

Submitted on 1 Jan 1989

HAL is a multi-disciplinary open access archive for the deposit and dissemination of scientific research documents, whether they are published or not. The documents may come from teaching and research institutions in France or abroad, or from public or private research centers.
L'archive ouverte pluridisciplinaire HAL, est destinée au dépôt et à la diffusion de documents scientifiques de niveau recherche, publiés ou non, émanant des établissements d'enseignement et de recherche français ou étrangers, des laboratoires publics ou privés. 


\title{
Limite de la région quasi neutre dans un semiconducteur
}

\author{
Z. T. Kuznicki $\left({ }^{1, *}\right)$ and B. Zimmermann $\left({ }^{2}\right)$ \\ (1) Laboratoire d'Automatique et d'Analyse des Systèmes du C.N.R.S., 7 avenue du Colonel Roche, \\ F-31077 Toulouse Cedex, France \\ (2) Institut Interdépartemental de Microélectronique et d'Optoélectronique de l'Ecole Polytechnique \\ Fédérale de Lausanne, CH-1015 Lausanne, Suisse
}

(Reçu le 25 avril 1988, révisé le 23 septembre 1988 et le 14 avril 1989, accepté le 30 mai 1989)

\begin{abstract}
Résumé. - Nous proposons une définition généralisée de la limite de la région quasi neutre du semiconducteur contenant une zone d'accumulation. Cette limite est fixée à l'endroit où deux facteurs macroscopiques de perturbation électrique sont égaux. Ainsi la région quasi neutre garde toujours les mêmes propriétés électriques, i. e. du poentiel macroscopique, du champ électrique interne, de la densité de charge, indépendamment du matériau et de l'intensité de la perturbation de la neutralité électrique du cristal. Les facteurs de perturbation sont déterminés dans le cadre d'une analyse approfondie de la loi électrostatique des interfaces abruptes dans les semiconducteurs non dégénérés à l'état d'équilibre thermodynamique. Ils n'ont pas d'interprétation physique simple. L'un d'eux caractérise la perturbation électrique en fonction des propriétés du semiconducteur considéré, l'autre facteur est tout à fait indépendant de ces propriétés. Notre définition est du type macroscopique. Elle est déterminée dans le cadre de la statistique de MaxwellBoltzmann, et a pour but de remplacer toutes les définitions plus ou moins arbitraires de l'épaisseur de la zone d'accumulation, jusqu'à présent couramment utilisées dans la modélisation ou simulation des dispositifs microélectronique. Elle peut être facilement généralisée à n'importe quelle perturbation électrique (statique ou dynamique) provoquant l'accumulation des porteurs libres.
\end{abstract}

\begin{abstract}
We propose a general definition of the boundary of the quasi-neutral region in a semiconductor with accumulation layer. This boundary occurs at the point where two electrical perturbation factors are equal. Thus the quasi-neutral region always keeps the same electrical properties, i.e. macroscopic potential, internal electric field, and charge density, independently of the electrical neutrality perturbation intensity. These perturbation factors, determined from a detailed analysis of the electrostatic law of abrupt interfaces in nondegenerate semiconductors at thermal equilibrium, have no simple physical interpretation. One of these factors characterizes the electrical perturbation in terms of the properties of the semiconductors while the second is totally independent of these properties. Our definition, formulated within the Maxwell-Boltzmann statistics, is of the macroscopic type. It is meant to replace other more or less arbitrary definitions of the thickness of the accumulated space charge used up to now in modelization and simulation of microelectronic devices. It could be easily generalized for other electrical perturbations (static or dynamic) that leads to an accumulation of free carriers.
\end{abstract}

\section{Introduction.}

L'effet d'une perturbation de la neutralité électrique d'un cristal semiconducteur se manifeste par la présence d'une charge d'espace qui peut être consti-

(*) A présent : 1) Université Louis Pasteur ; département de Physique, 3, rue de l'Université ; F-67000 Strasbourg Cedex, France.

2) Centre de Recherches Nucléaires (IN2P3), laboratoire PHASE (UA du CNRS N²92), F-67037 Strasbourg Cedex, France. tuée soit de porteurs libres excédentaires (couche d'accumulation) soit d'ions dopants non compensés (couche de dépeuplement).

Une telle perturbation peut être créée soit de façon extérieure, par application d'une tension de grille (structure MOS ou MIS), soit de manière intrinsèque, par suite de différences dans les structures de bande des matériaux constituants (hétérojonctions) ou dans le type et taux de dopage (homojonctions).

Les premières études expérimentales de contrôle 
et de variations de l'épaisseur effective de la couche d'accumulation ont été faites sur des MOS au Silicium. Ces structures ont l'avantage de permettre de larges variations de la densité de porteurs par simple application d'une tension de grille adéquate. Mais les mêmes couches d'accumulation sont aussi présentes dans certaines homo- et hétérojonctions où, dans l'état stationnaire, elles ont un caractère intrinsèque, déterminé uniquement par la configuration et les propriétés des matériaux constituants.

L'extension de la charge d'espace des porteurs libres est généralement très faible. C'est pourquoi on assimile habituellement une couche d'accumulation à une « distribution superficielle » de charge. Il est vrai que cette approche ne néglige qu'un très faible pourcentage de la charge totale (pratiquement quelques pour cent). Toutefois cette petite partie de charge d'espace est répartie dans un volume de cristal important.

C'est pourquoi dans le cadre du traitement macroscopique, l'épaisseur de la couche d'accumulation dans les cristaux semiconducteurs était déterminée jusqu'à présent de façon plus ou moins arbitraire ; ce qui conduit à des dispersions importantes, pouvant atteindre plusieurs ordres de grandeur.

Dans l'électronique moderne la région non perturbée ou quasi neutre devrait avoir une définition unique et être déterminée avec une grande précision. On imagine mal sur quelle base on peut poser une définition générale de la région électriquement neutre ou quasi neutre sans avoir une référence physique universelle.

Sur la figure 1 nous donnons un recueil des définitions parues dans la littérature. Pour cela nous avons représenté une couche d'accumulation dans le cas d'une forte perturbation stationnaire (potentiel électrostatique normalisé sur l'unité thermodynamique, $U_{\mathrm{s}}=13,0$; champ électrique maximal normalisé sur l'unité thermodynamique et le rayon de Debye extrinsèque, $F_{\mathrm{s}}=940$ ).

Avec une telle approche (illustrée sur la figure 1), la couche d'accumulation perd facilement son sens numérique. Ce qui conduit à un nombre assez important d'imprécisions où même d'erreurs parues un peu partout dans la littérature. Citons par exemple la publication relativement récente de Jindal et Warner [5] où les auteurs évoquent le rôle du rayon de Debye extrinsèque comme une longueur universelle de normalisation dans le cas des couches d'accumulation. Comme nous le verrons ci-dessous (dans le chapitre «Application au cas...») ceci ne pourrait être vrai qu'à partir d'une certaine intensité de la perturbation $\left(F_{\mathrm{s}}>7,0\right)$.

Dans cet article nous proposons d'introduire une définition universelle de l'épaisseur de la zone de charge d'espace constituée des porteurs libres. Elle est basée sur un développement très simple de la formule exacte de la concentration des porteurs

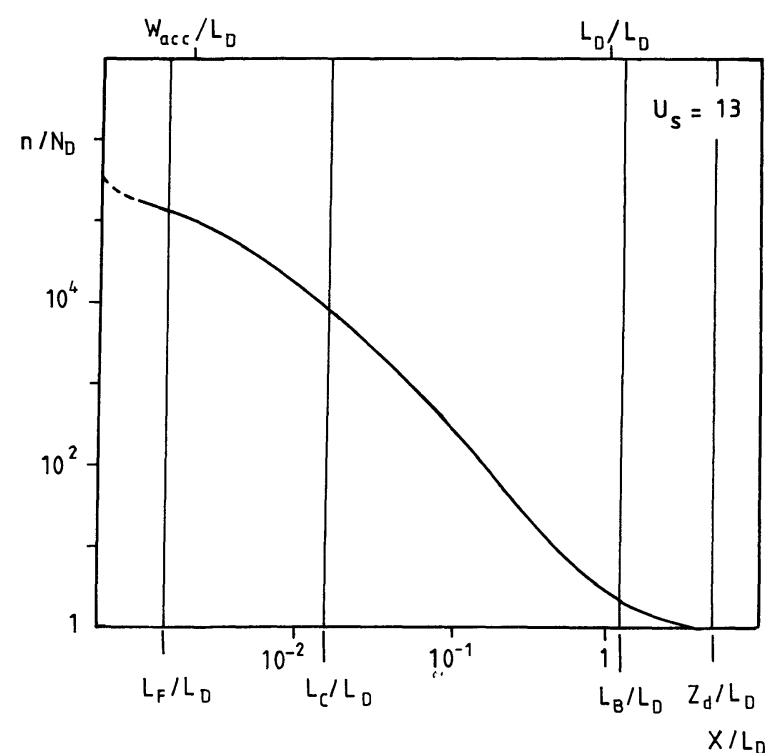

Fig. 1. - Répartition des porteurs excédentaires dans une couche d'accumulation dans le cas d'une perturbation intense. On a porté les distances caractéristiques calculées selon les différentes définitions de la charge d'espace : $z_{\mathrm{d}}$ est la distance de courbure des bandes d'énergie à proximité de la surface $[1,2] ; L_{\mathrm{D}}$ est le rayon de Debye extrinsèque utilisé couramment comme une distance de normalisation, cf. [3-6]; $L_{\mathrm{c}}$ est l'épaisseur effective de la zone de charge d'espace mesurée entre la surface et le centre de la zone de charge d'espace $[7,8] ; L_{\mathrm{F}}$ est le rayon d'écrantage sur lequel la concentration des porteurs excédentaires diminue d'un facteur $e$ (la base de logarithme néperien) [8-10]; $w_{\text {acc }}$ est l'épaisseur de la couche d'accumulation [11].

[Excess carrier distribution in an accumulation layer in the case of a strong perturbation. The drawn characteristic lengths have been computed in accordance with the different space charge definitions : $z_{\mathrm{d}}$ energy band bending length in the surface neighborhood $[1,2] ; L_{\mathrm{D}}$ extrinsic Debye length usually considered as an excellent choice for scaling the accumulation curves, i.e. [3-6]; $L_{c}$ effective charge distance measured from the surface to the centre of the space charge $[7,8] ; L_{\mathrm{F}}$ screening radius along which the excess carrier concentration decreases by $1 / e(e$ is the base of the Naperian logarithm) [8-10] ; $w_{\text {acc }}$ accumulation layer thickness [11].]

libres à l'interface d'une homojonction abrupte L-H (ang. «Lightly-Heavely » doped regions : $n^{+}-n$, $\left.p^{+}-p\right)[12,13]$.

Nous montrons que la concentration des porteurs libres est en effet un produit de deux facteurs (appelés ici facteurs de perturbation électrique), qui ont une variation totalement différente l'une de l'autre en fonction de la distance de l'interface ainsi qu'en fonction de l'intensité de la perturbation.

L'endroit où ces deux facteurs sont égaux marque la limite de la région quasi neutre. Ainsi la région quasi neutre garde toujours les mêmes propriétés électriques (i.e. du potentiel macroscopique, du 
champ électrique interne, de la densité de charge), indépendamment du matériau et de l'intensité de la perturbation de la neutralité électrique du cristal.

Il se trouve qu'en appliquant notre définition, le bord de la région quasi neutre est toujours fixé là où la concentration des porteurs libres $n\left(L_{\mathrm{B}}\right)$ est 2,43793 fois plus importante que celle d'équilibre, ce qui correspond au potentiel électrostatique normalisé $U\left(L_{\mathrm{B}}\right)=0,89115{ }^{(1)}$. Le volume de la région quasi neutre devient ainsi une fonction de l'intensité de la perturbation «appliquée " (respectivement intrinsèque ou extrinsèque).

En deuxième partie de cet article, nous illustrons l'implication de notre définition sur le déplacement de la limite de la région quasi neutre dans les homojonctions abruptes $n^{+}-n^{-}$de GaAs en fonction du rapport de taux de dopage. Dans nos exemples, la couche faiblement dopée représente le semiconducteur en question, alors que la couche fortement dopée ne fait pas partie du système en soi, mais cause la perturbation électrique.

Dans ces structures, la limite de la région quasi neutre, telle qu'elle est définie plus haut, peut bien sûr être décelée numériquement. C'est ce que nous avons fait afin de comparer la limite obtenue numériquement à celle ressortant de nos considérations analytiques.

\section{Limite de la région quasi neutre.}

La quasi-neutralité électrique correspond à une perturbation appliquée au cristal si peu intense qu'elle modifie essentiellement la répartition des porteurs sur les niveaux de leurs bandes d'énergie, n'influençant pratiquement pas le nombre de porteurs libres. Elle se caractérise par une densité nette de charge très faible vis-à-vis de celle qui est liée aux seules impuretés. Les résultats des calculs des propriétés électriques effectués dans l'hypothèse de neutralité stricte sont universellement utilisés avec d'autant plus de précision qu'on approche de la situation de neutralité totale.

Le potentiel dans une zone quasi neutre peut en effet varier en fonction de la distance dans d'assez larges limites bien que la densité nette de charge conserve des valeurs négligeables.

Nous admettons l'hypothèse que la limite entre la région quasi neutre et la région chargée se trouve à l'endroit où les deux facteurs de perturbation (un lié directement aux propriétés du cristal et l'autre totalement indépendant de ces propriétés) sont égaux :

(1) En effet, une définition semblable a déjà été proposée par Gunn [14] qui a établi que cette limite se trouve là où le potentiel électrostatique normalisé est égal à 1,0 . Ceci est la variation maximale que ce potentiel peut atteindre dans la région la plus dopée de la jonction L-H.

$$
n_{\text {mi }}\left(L_{\mathrm{B}}\right)=n_{\mathrm{me}}\left(L_{\mathrm{B}}\right)
$$

où $L_{\mathrm{B}}$ est la distance, mesurée à partir du maximum de la perturbation électrique présente dans le cristal, désignant la limite entre la région quasi neutre et la région chargée.

La définition de $n_{\mathrm{mi}}\left(L_{\mathrm{B}}\right)$ et $n_{\mathrm{me}}\left(L_{\mathrm{B}}\right)$ ressortira de ce qui suit dans le paragraphe "Solution analytique ».

\section{Facteurs de perturbation.}

A l'équilibre thermodynamique l'intégration rigoureuse de l'équation de Poisson, étendue à toute la région semiconductrice perturbée, permet de développer la formule exacte liant la concentration des porteurs excédentaires au champ électrique. Ceci est vrai en n'importe quel point de la région perturbée (v. loi électrostatique des interfaces abruptes d'homostructures semiconductrices [13]). Dans le cas unidimensionnel la loi électrostatique s'écrit :

$$
\frac{n(x)}{N_{\mathrm{D}}}-\ln \frac{n(x)}{N_{\mathrm{D}}}=0,5 F^{2}(x)+1,0
$$

où

$x \quad$ est la distance mesurée par rapport à l'interface (surface) ;

$N_{\text {D }}$ est la concentration de dopant non compensé ;

$F(x)$ est le champ électrique normalisé sur le rayon de Debye extrinsèque $\left(L_{\mathrm{D}}\right)$ et l'unité thermodynamique $\left(U_{\mathrm{T}}=k T / q\right)$.

Après quelques opérations mathématiques élémentaires, nous pouvons exprimer la concentration des porteurs excédentaires normalisée, $n(x) / N_{\mathrm{D}}$, sous la forme d'un produit de deux facteurs de natures physiques différentes [15]

$$
\frac{n(x)}{N_{\mathrm{D}}}=n_{\mathrm{mi}}\left(F_{\mathrm{s}}, x\right) n_{\mathrm{me}}\left(F_{\mathrm{s}}, x\right) \text {; }
$$

où

$$
\begin{aligned}
& n_{\mathrm{mi}}=N^{(1 /(N-1))} \\
& n_{\mathrm{me}}=N / e
\end{aligned}
$$

$N$ est une fonction qui dépend de l'intensité de la perturbation et varie dans l'espace géométrique; cette fonction dépend alors de champ électrique local (voir Eq. (6)) ;

$e \quad$ est la base de logarithme népérien ;

$F_{\mathrm{s}}$ est la valeur du champ électrique normalisé à l'interface.

Ici, $n_{\mathrm{mi}}$ représente le facteur décrivant la perturbation peu intense de la neutralité électrique, restant toujours en relation avec les propriétés du cristal contenant la charge d'espace. Sa valeur correspond à la concentration d'une fraction des porteurs excédentaires gardant la même température électronique que celle du réseau. Par contre $n_{\text {me }}$ renferme les causes externes aux propriétés du cristal considéré, 
se manifestant surtout dans les perturbations intenses. Sa valeur correspond à la somme des fractions de la concentration excédentaire refroidies électroniquement $[16,17]$. Les fractions évoquées ci-dessus [17] correspondent directement aux fractions décrites, entre autres, par Stern dans le cas des puits quantiques accolés aux surfaces ou interfaces des cristaux semiconducteurs localement perturbés [18, 19].

Remarque: Dans le cas particulier d'une couche d'accumulation d'homojonction abrupte $L-H\left(n^{+}-n\right.$ ou $\left.p^{+}-p\right)$, pris comme exemple dans nos calculs, la fonction $N$ a le sens physique du rapport des taux de dopage et l'équation (5) correspond directement à l'équation développée par Chandra [20]. Ceci est vrai pour les perturbations très élevées (perturbations représentées dans le cas de la jonction $L-H$ par ce même rapport des taux de dopage).

$\mathrm{Au}$ fur et à mesure que l'intensité de perturbation est accrue le rôle de $n_{\mathrm{mi}}$ devient négligeable $\left(n_{\mathrm{mi}} \rightarrow 1,0\right.$ quand $N \rightarrow \infty$ ) tandis que $n_{\text {me }}$ croît systématiquement, suivant de plus en plus près la concentration excédentaire normalisée, figures 2 et 3. Ceci veut dire que pour les perturbations intenses, les propriétés électriques du cristal (dopage, conductivité) n'ont aucune influence sur la charge d'espace des porteurs accumulés.

En général, la fonction $N(F, x)$ est liée au champ électrique (intrinsèque ou provoqué par la tension de grille) de la manière suivante, déduite de la loi électrostatique $[12,13]$

$$
F=\sqrt{2(M / e-\ln M)}
$$

où

$$
M=N^{N /(N-1)} .
$$

Sur les figures 2 et 3 nous rapportons les variations de $N$ en fonction de $x$ et $F_{\mathrm{s}}$, gardant une fois $x$ égal à 0 (Fig. 2) et l'autre fois $F_{\mathrm{s}}$ égal à 8,23246 (Fig. 3).

La figure 3 montre aussi les variations spatiales des deux facteurs, $n_{\mathrm{mi}}$ et $n_{\mathrm{me}}$, ainsi que la variation

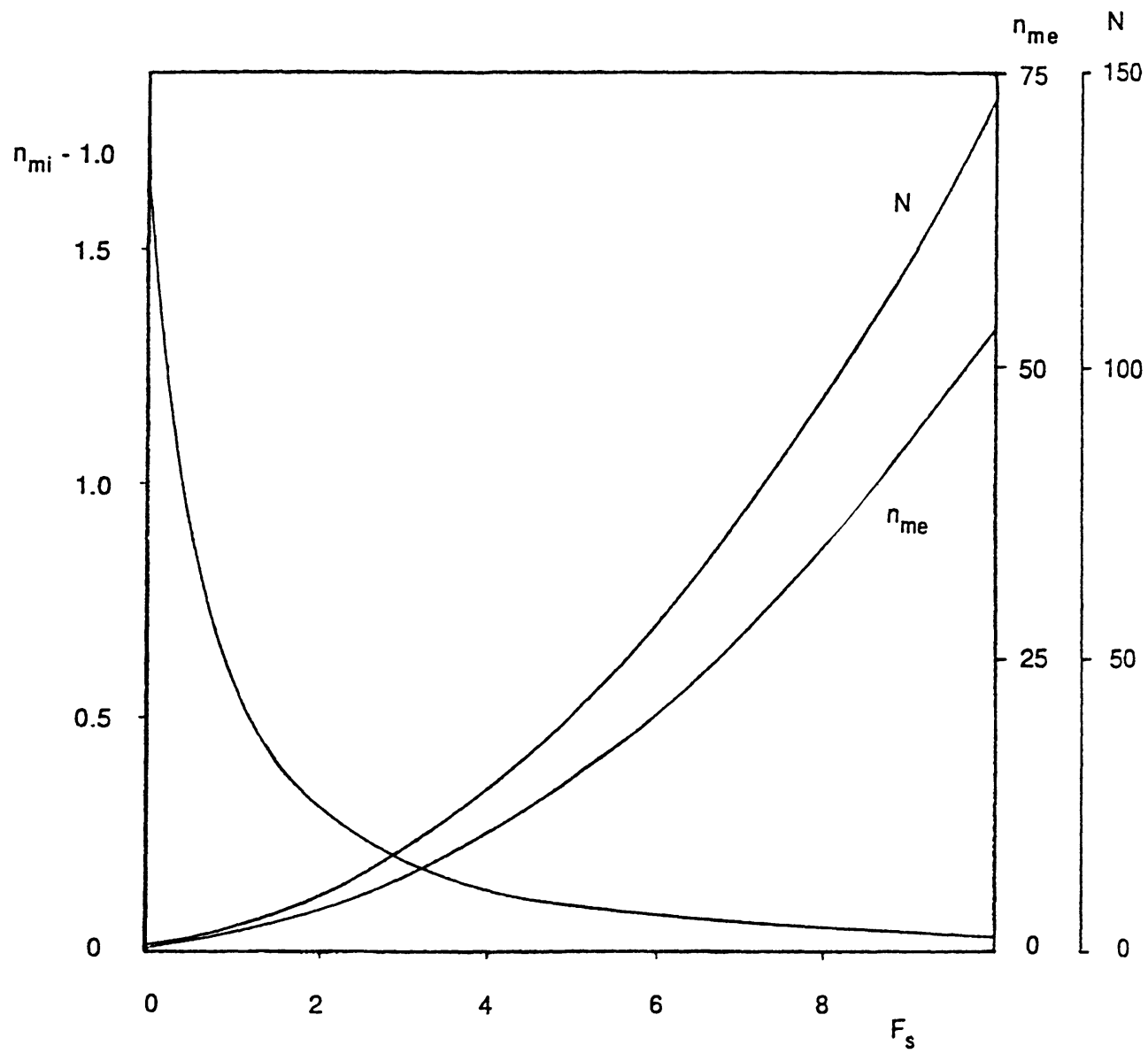

Fig. 2. - Variation de la fonction $N$ ainsi que de deux facteurs de perturbation en fonction du champ électrique maximal normalisé, $F_{\mathrm{s}} ; n_{\mathrm{mi}}$ est le facteur de perturbation de la neutralité électrique lié aux propriétés intrinsèques du cristal ; $n_{\mathrm{mc}}$ est le facteur de perturbation de la neutralité électrique indépendant des propriétés du cristal.

[The $N$ function as well as two perturbation factor distributions versus normalized maximal electric field, $F_{\mathrm{s}} ; n_{\mathrm{mi}}$ electrical neutrality perturbation factor related to the intrinsic semiconductor properties; $n_{\mathrm{me}}$ electrical neutrality perturbation factor independent of the crystal properties.] 


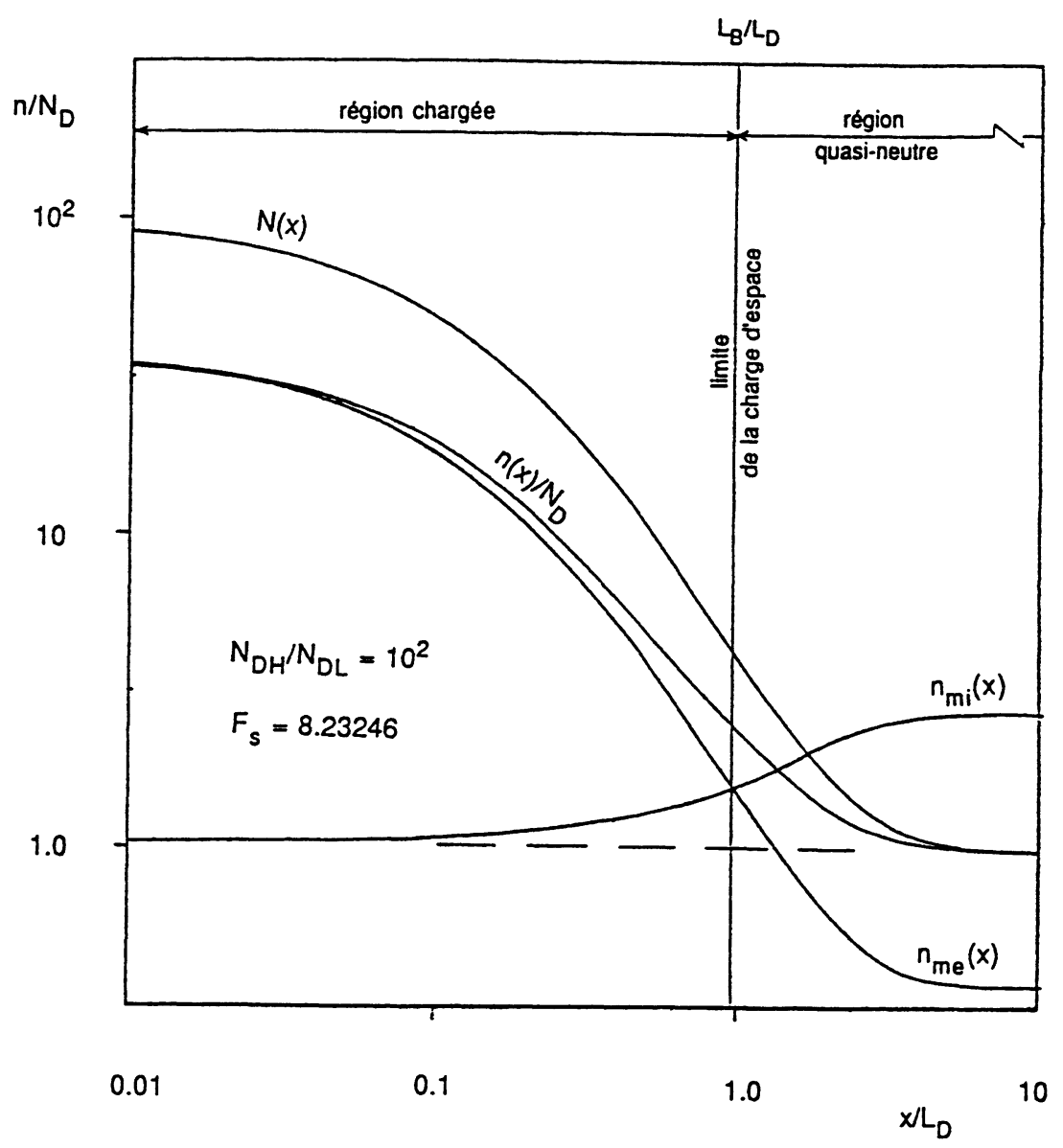

Fig. 3. - Variations spatiales des facteurs de perturbation, $n_{\mathrm{mi}}$ et $n_{\mathrm{me}}$, de la fonction $N$ et de la concentration des porteurs excédentaires $n(x) / N_{\mathrm{D}}$ dans une couche d'accumulation correspondant à une perturbation d'intensité moyenne $\left(F_{\mathrm{s}}=8,23246\right)$.

[Space distributions of the : perturbation factors $n_{\mathrm{mi}}$ and $n_{\mathrm{me}}$; function $N$ and excess carrier concentration $n(x) / N_{\mathrm{D}}$, respectively, in an accumulation layer, in the case of the medium strength perturbation $\left(F_{\mathrm{s}}=10\right)$.]

spatiale de la concentration respective des porteurs excédentaires, dans une couche d'accumulation où le champ électrique maximal normalisé $F_{\mathrm{s}}$ est égale à $8,23246$ (ce qui correspond à $N=100)\left({ }^{2}\right)$. Ceci concerne n'importe quelle zone d'accumulation : soit d'une jonction abrupte L-H, soit d'une structure MOS.

Nous pouvons directement tracer les variations des $n_{\mathrm{mi}}$ et $n_{\mathrm{me}}$ et fixer la limite de la région quasi neutre dans l'espace de la fonction $N$, représentant ainsi la perturbation (Eqs. (6) et (7)), à partir des équations (4) et (5), figure 4.

\section{Application au cas de l'homojonction abrupte L-H.}

Pour étudier les variations spatiales de $n_{\mathrm{mi}}$ et $n_{\text {me }}$, il faut connaître la variation spatiale du potentiel électrostatique. Ainsi on est amené à résoudre l'équation de Poisson dans l'espace géométrique, ce

( $\left.{ }^{2}\right)$ Le détail de calculs est présenté ci-dessous dans le chapitre "Solution analytique ». qui n'est pas un problème trivial (même dans le cas unidimensionnel) et requiert habituellement soit des approximations analytiques, soit des moyens de calcul numérique.

Ehlers et Mills ont publié récemment [21] une étude microscopique, très détaillée, concernant la couche d'accumulation et de déplétion, sous très faible perturbation.

La variation du potentiel électrostatique normalisé, obtenue par ces auteurs (Fig. 2 dans le [21]) grâce à leur modèle microscopique est en parfait accord avec les résultats de notre modèle macroscopiques, obtenus dans le cadre de la solution généralisée de l'équation de Poisson [16, 17].

La figure 5, partiellement tirée de [21], rapporte la comparaison entre les répartitions du potentiel électrostatique normalisé obtenues par Ehlers et Mills et par nous. Dans ce cas notre fonction de perturbation $N$ prend la valeur de 6,25 , ce qui correspond à une perturbation peu intense. Ceci se traduit par une forte contribution de la fraction de population des porteurs en excès $(\approx 65 \%)$, gardant toujours 


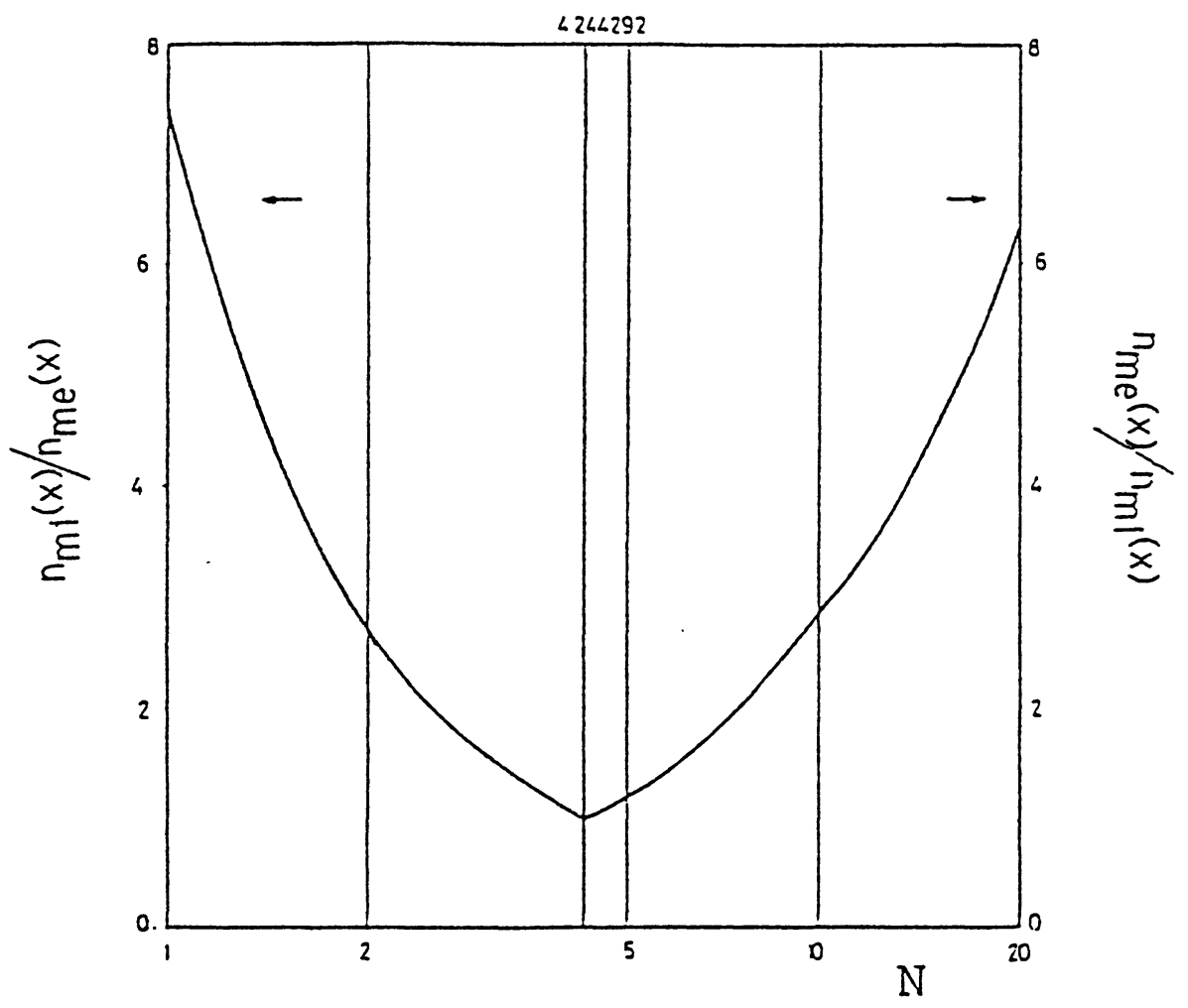

Fig. 4. - Variation des rapports des deux facteurs de perturbation dans les jonctions abruptes L-H en fonction du rapport des taux de dopage $N[15]$ : définition de la limite de région quasi neutre, $N=4,244292$.

[Two perturbation factor ratio distributions versus doping ratio function $N$ in the L-H junction [15] ; quasi-neutrality region boundary definition, $N=4.244292$.]

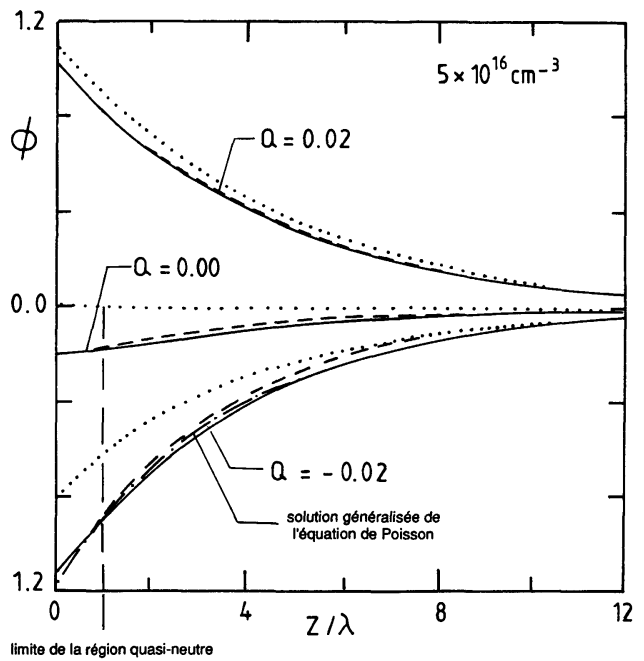

Fig. 5. - Variation des potentiels publiés par Ehlers et Mills (Fig. 2 dans [21]). Le résultat obtenu dans le cadre de la solution généralisée de l'équation de Poisson [17] est introduit sous la forme d'une courbe en tirés-points. Dans nos calculs on a adopté la valeur de $N=6,25$ (Eqs. (3)(7)). La ligne verticale en tirés représente la limite de la région quasi neutre.

[Potential distributions published by Ehlers and Mills (Fig. 2 in [21]). Result of the general Poisson's equation solution has been introduced as the dot-dashed curve. In our calculation we have taken that $N=6.25$ [17]. The limit to the quasi-neutral region is shown by the vertical dashed line.] l'isotropie de son agitation thermique [17] (Fig. 6). De plus la population avec une agitation peu anisotrope est de l'ordre de $13 \%$.

La légère différence entre les résultats du modèle « microscopique » d'une part et du modèle " macroscopique " d'autre part provient du fait que les auteurs de [21] prennent en compte la valeur moyennée du champ électrique, alors que nous considérons le champ local en chaque point.

Nous avons effectué nos calculs afin d'avoir le point d'intersection des deux courbes à la limite de la région quasi neutre (la ligne droite verticale en tirés sur la figure 5). A cette fin nous avons procédé de façon suivante. Tout d'abord nous avons déterminé la distance entre la surface et la limite de la région quasi neutre sur la figure 2 dans le [21] (ne prenant en compte que la variation de potentiel auto-compatible ainsi publié). Puis, ayant $L_{\mathrm{B}}$, nous sommes en mesure de déterminer la valeur de $N$ et recalculer notre variation du potentiel à l'aide de la solution généralisée de l'équation de Poisson [17].

Nous pouvons ainsi comparer la perturbation traitée par Ehlers et Mills [21] avec une gamme pratiquement complète d'intensités des perturbations électriques réellement envisageables, figure 6.

Comme à la limite de la région quasi neutre la perturbation est par définition, très réduite, on peut 

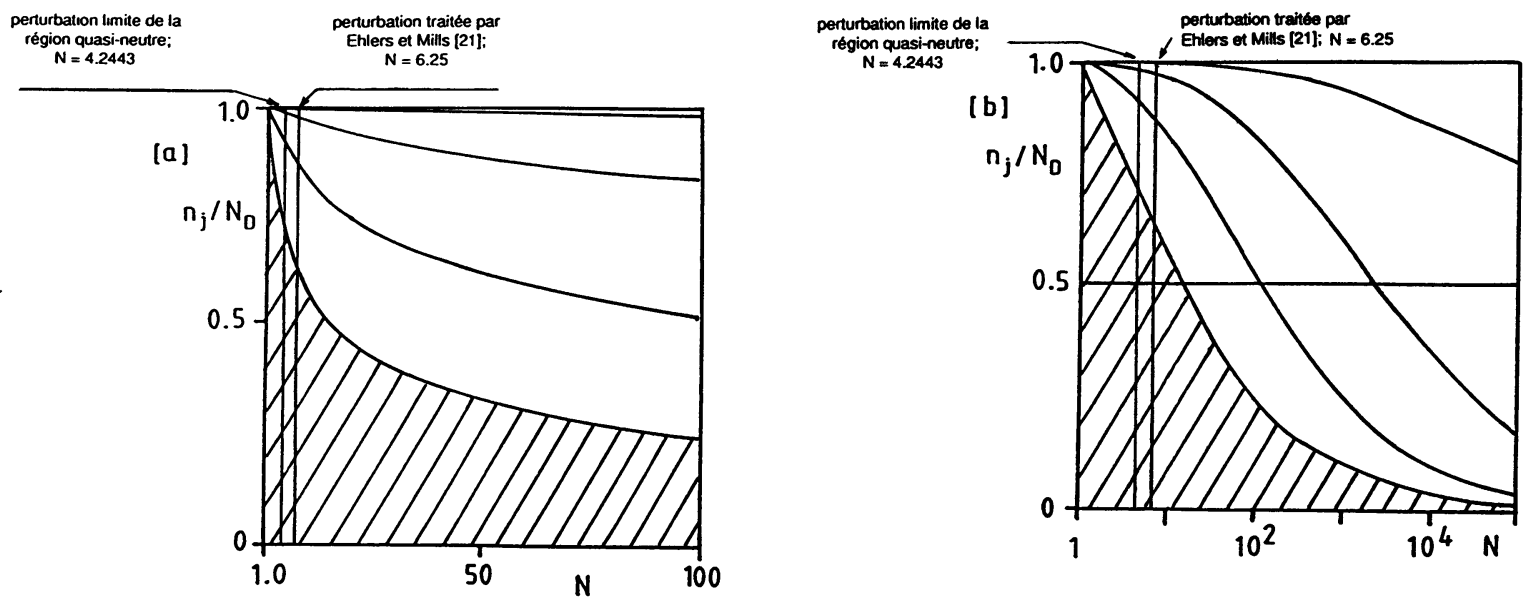

Fig. 6. - Répartitions de populations excédentaires relatives d'agitations anisotropes [17] en fonction de l'intensité de perturbation représentée par la fonction $N$ : a) coordonnées linéaires, intensités moyennes ; b) coordonnées semilogarithmiques, toute la gamme des intensités réellement envisageables. La zone hachurée représente la fraction excédentaire conservant l'isotropie de l'agitation thermique.

[Distributions of anisotropic oscillation fractions of carriers versus perturbation intensity function $N$ : a) linear coordinates ; b) semi-log coordinates, entire really permitted perturbation intensity range [17]. Dashed part shows the excess carrier fraction of isotropic oscillations.]

se contenter ici de la statistique de MaxwellBoltzmann.

Néanmoins dans le cas de fortes intensités de perturbation du côté de la surface il y a un risque de déviation de la variation calculée par rapport à sa valeur réelle. Il faut donc reprendre la statistique de Fermi-Dirac. Heureusement, comme nous pouvons le voir sur la figure 1 par exemple, l'extension effective de la charge d'une couche d'accumulation est généralement si faible, qu'une erreur éventuelle provenant de la distribution de Maxwell-Boltzmann, admise dans notre cas de solution analytique, est tout à fait négligeable. C'est pourquoi beaucoup d'auteurs assimilent une couche d'accumulation produite par une perturbation intense à une distribution superficielle de charge.

L'erreur de l'approche simplifiée, effectuée sur la base de la statistique de Maxwell-Boltzmann, est donc très limitée, étant donné le rapport de la distance de la limite de la région quasi neutre et de l'épaisseur effective de couche d'accumulation d'une perturbation intense. Ce rapport est de l'ordre : de 45, quand le maximum du champ électrique normalisé, $F_{\mathrm{s}}$ est égale à 75 et de $\mathbf{2 3 0}$ pour $F_{\mathrm{s}}$ égale à 85 . Bien entendu, pour les perturbations moyennes $\left(F_{\mathrm{s}}<40\right)$, et surtout pour les perturbations faibles, la statistique de Maxwell-Boltzmann est applicable dans toute la région perturbée avec une erreur très réduite.

Dans le cas de perturbations intenses les deux phénomènes opposés, à savoir l'accumulation et la déplétion, ne peuvent pas être traités ensemble. La déplétion reste toujours liée aux propriétés du cristal, plus précisément au taux de dopage, tandis que l'accumulation devient totalement indépendante de celles-ci.
A partir d'une analyse macroscopique, prenant en compte la variation de la densité de «charge d'espace modèle " d'une homojonction abrupte (L$\mathrm{H}$ ), nous sommes en mesure de déterminer plusieurs longueurs caractéristiques dont une joue le rôle de la distance entre la surface (le maximum de l'intensité de perturbation) et la limite de la région quasi neutre.

Comme exemple de calculs nous avons donc choisi l'homojonction abrupte L-H $\left(n^{+}-n\right)$, où nous avons procédé selon deux méthodes complémentaires :

- une méthode analytique généralisée ; approximation simplificatrice qui permet d'étudier l'état électrique du cristal par analyse de comportement de quelques fractions de porteurs libres excédentaires; cette représentation approximative enveloppe tous les cas pratiques avec une exactitude frôlant la perfection dans le cadre de la statistique de MaxwellBoltzmann [16, 17] ;

- une méthode numérique auto-compatible [2224]; pour vérifier, compléter et généraliser nos résultats, dits ainsi « analytiques », nous avons utilisé une méthode numérique d'éléments finis [24]: d'abord dans l'hypothèse de la statistique de Maxwell-Boltzmann et de l'ionisation totale des impuretés (ce qui est admis dans nos calculs analytiques); puis dans l'hypothèse de la statistique de FermiDirac et de l'ionisation partielle des impuretés.

SOLUTION ANALYTIQUE. - L'avantage de cette méthode réside dans le fait qu'elle permet de traiter le problème analytiquement (une fois la solution numérique adéquate trouvée, celle-ci représente une base de données pour tous les cas réellement envisageables). 
La solution de type analytique permet de déduire la formule reliant l'épaisseur de la zone de charge d'espace, normalisée sur le rayon de Debye extrinsèque, à l'intensité de perturbation (représentée ici par le potentiel macroscopique); celle-ci est donnée sous la forme :

$$
L_{\mathrm{B}} / L_{\mathrm{D}}=\ln \left(U_{\mathrm{D}} / U\left(L_{\mathrm{B}}\right)-\sum_{j}^{m-1} U_{j} \exp \left(-L_{\mathrm{B}} / L_{j}\right)\right)
$$

où

$U\left(L_{\mathrm{B}}\right) \quad 0,89115$ est une valeur constante du potentiel macroscopique normalisé à la limite de la région quasi neutre, déterminée une fois pour toutes grâce à l'équation (1); elle correspond au champ électrique $F=1,04574$ et la concentration excédentaire $n(x) / N_{\mathrm{D}}=1,43793$;

$U_{\mathrm{D}} \quad$ est une composante de potentiel macroscopique liée à la fraction de porteurs excédentaires ayant l'agitation thermique isotropique [17] ;

$U_{j}$ et $L_{j}$ sont respectivement : une composante de potentiel macroscopique et une distance caractéristique liées à la j-ème fraction de porteurs excédentaires se distinguant par une agitation thermique anisotropique [16, 17] ;

$m$

est un nombre de fractions des porteurs excédentaires (en général $m=5$ [17]).

Malheureusement cette formule ne peut être exprimée que sous forme de variables non séparables ; la résolution n'est donc pas triviale et exige des traitements numériques adéquats. Nous avons utilisé à cette fin une méthode de solutions numériques des systèmes de plusieurs équations non linéaires de la bibliothèque d'IBM afin de trouver des valeurs du jeu de plusieurs paramètres caractéristiques [16, 17]. L'exemple de nos résultats ainsi calculés est représenté sur les figures 3 et 5 .

Sur la base de représentation analytique [17] (Eq. (8)) nous sommes en mesure de donner une interprétation physique aux facteurs de perturbations :

- le facteur extrinsèque $n_{\mathrm{mi}}$ correspond à une fraction de concentration des porteurs libres en excès gardant l'agitation thermique isotrope (décrite dans (8) par la composnte $U_{\mathrm{D}}$ de potentiel normalisé) ;

- le facteur extrinsèque $n_{\mathrm{me}}$ représente $m-1$ (en général égale à quatre) fractions de concentration des porteurs libres en excès se caractérisant par une anisotropie de l'agitation thermique. Cette anisotropie correspond au phénomène appelé aussi refroidissement électronique [16].
SOLUTION NUMÉRIQUE. - On obtient les mêmes résultats par des méthodes exclusivement numériques. A cette fin nous résolvons l'équation de Poisson à l'aide de la méthode des éléments finis, utilisant des fonctions de base triangulaires [24]. Le maillage de l'espace unidimensionnel $x$ est irrégulier et tel que la variation du potentiel entre deux points consécutifs ne dépasse pas $0.3 \mathrm{meV}$.

Comme conditions aux bords, nous imposons que le champ électrique soit nul aux bords de toute la structure, qui cette fois-ci (jonction L-H) inclut aussi la région perturbatrice, à savoir la plus dopée. Ceci (dans notre cas unidimensionnel et selon la loi de Gauss) implique la neutralité globale de la structure considérée. Notons que les calculs numériques sont exécutés pour la structure totale d'une jonction L-H abrupte qui consiste en une couche fortement dopée et une couche faiblement dopée, séparées, l'une de l'autre par une zone de transition de dopage d'une longueur infinitésimale.

La densité de charge est évalué soit selon la statistique de Fermi-Dirac, soit selon la statistique de Maxwell-Boltzmann. Ceci dans le but de contrôler l'erreur introduite par la statistique de MaxwellBoltzmann. Le taux d'ionisation des dopants peut être soit évalué selon la statistique choisie, soit (afin de permettre une meilleure comparaison avec le calcul analytique) être supposé total.

L'évaluation de la densité de charge selon la statistique de Maxwell-Boltzmann et en supposant une ionisation complète des dopants, comme c'est aussi utilisé dans nos calculs analytiques, permet ainsi mieux comparer les résultats analytiques et numériques. Il est généralement admis (par exemple, dans [21]) que la répartition des impuretés ionisées dans un semiconducteur non dégénéré reste homogène jusqu'a la surface.

L'application de la statistique de Fermi-Dirac pour les densités d'électrons, de trous et de dopants, décrit mieux la réalité physique. Il est intéressant de voir si les mêmes observations qualitatives et quantitatives sont faites avec les deux évaluations de la densité de charge. Ceci a permis de mettre en évidence une grande exactitude de l'approche analytique dont sortent les deux facteurs de perturbation $n_{\text {mi }}$ et $n_{\text {me }}$.

Nous pouvons constater que dans un cristal non dégénéré les valeurs de $L_{\mathrm{B}}$ trouvées « analytiquement " et "numériquement » sont pratiquement les mêmes jusqu'aux perturbations intenses $\left(F_{\mathrm{s}}>40\right)$ ou extrêmement intenses $\left(F_{\mathrm{s}}>250\right)$, où $L_{\mathrm{B}} \rightarrow 1,2 L_{\mathrm{D}}$. la solution « analytique », ne prenant en compte que la statistique de Maxwell-Boltzmann, suit de très près la plus réaliste solution numérique, avec la statistique Fermi-Dirac et l'ionisation partielle, jusqu'à $F_{\mathrm{s}}=860$ (l'erreur relative estimée sur la base de la fonction $N$, Eq. (6), ne dépasse jamais $0,132 \%)$. 


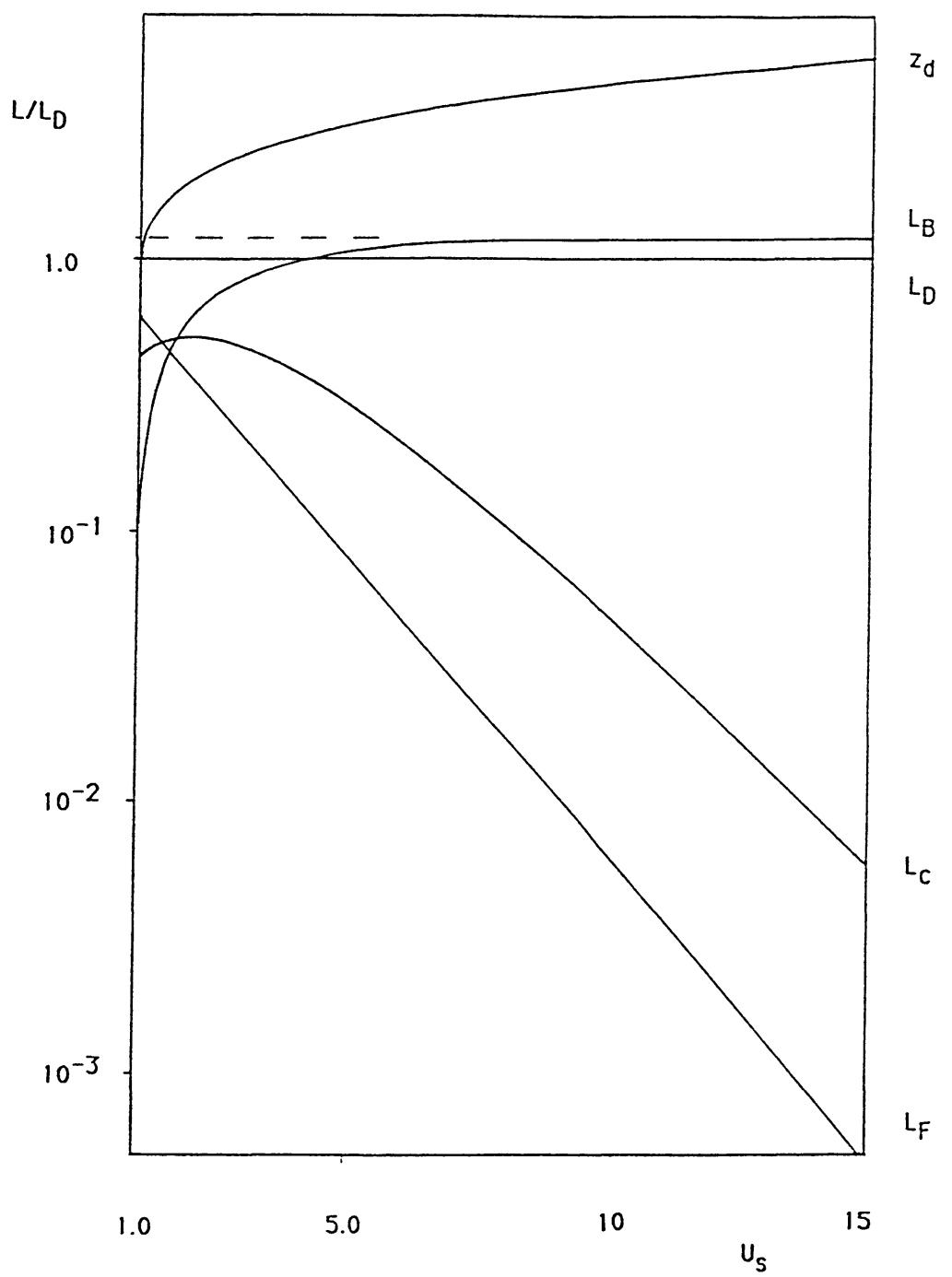

Fig. 7. - Variation des différentes distances caractéristiques de la charge d'accumulation en fonction du potentiel électrostatique maximal.

[Different characteristic length distribution versus maximal electrostatic potential.]

Remarque : Les valeurs de $F_{\mathrm{s}}$ citées ci-dessus correspondent aux calculs "analytiques », compte tenu de l'expression (5). La comparaison des résultats s'effectue sur la base de la fonction $N$, qui est uniforme dans les deux cas : " analytique » et « numérique ». En réalité les valeurs de champ électrique, $F_{\mathrm{s}}$, pour la même valeur de fonction $N$, sont différentes (cf. pour $N=10^{6}: F_{\text {s «analytique» }}=857,75$ et $\left.F_{\mathrm{s} \text { «numérique» }}=560,6\right)$. Le même calcul d'erreur relative sur la base du champ électrique ne dépasse jamais $0,3 \%$.

\section{Conclusions.}

Nous avons montré que la longueur $L_{\mathrm{B}}$ (définie par la formule (8)), déterminée sur la base de la loi électrostatique des homostructures semiconductrices abruptes dopées uniformément et non dégénérées, quelle que soit l'intensité de la perturbation de neutralité électrique, représente une caractéristique universelle, décrivant l'épaisseur de la charge d'espace d'accumulation des porteurs libres dans un cristal semiconducteur.

Ainsi, nous obtenons la limite séparant la région chargée de la région quasi neutre dans le cas des jonction $\mathrm{L}-\mathrm{H}$, définie en fonction du dopage, du champ électrique appliqué, etc. Cette limite partage le volume perturbé d'un cristal semiconducteur en deux parties dont une (la région quasi neutre) reste toujours la même du point de vue des propriétés électriques i.e. du potentiel macroscopique, du champ électrique interne, de la densité de charge d'espace. Ce ne sont que les deux volumes en question qui varient avec l'intensité de perturbation.

De plus notre définition de la limite de la région quasi neutre peut être facilement généralisée à n'importe quel système semiconducteur uniformément dopé, ainsi que à n'importe quelle perturbation électrique statique ou même dynamique, qui provoque l'accumulation des porteurs libres. 


\section{Bibliographie}

[1] VAN DER ZIEL A., Solid State Physical Electronics (Pretince-Hall, Inc., Englewood Cliffs, New Jersey, USA) 1976.

[2] BeEREnS J., Thèse de doctorat, Université de Sherbrooke, Québec, Canada (1986).

[3] Goldberg C., Solid-State Electron. 7 (1964) 593600.

[4] Hauser J. R., Littlejohn M. A., Solid-State Electron. 11 (1968) 667-674.

[5] Jindal R. P., WARNeR R. M. Jr., IEEE Trans. Electron. Dev. ED-29 (1982) 1944-1945.

[6] Warner R. M. Jr., GRUNG B. L., Transistors ; Fundamentals for the Integrated-Circuit Engineer (Wiley, New York) 1983.

[7] Many A., Goldstein Y., Grover N. B., Semiconductor Surfaces (North-Holland, Amsterdam) 1965.

[8] Many A., Surface Science. Lectures Presented at an International Course ; Pt. I, Trieste, Italy, 16 January-10 April 1974 ; Vienna, Austria : IAEA 1975, pp. 447-500.

[9] Kuznicki Z. T., Propriétés Electriques des Couches Minces Polycristallines; Ed. PWN WarszawaLodz, 1984 (en Polonais); Some Analytical Descriptions of Abrupt Semiconductor L-H Type Structures in Stationary State ; Rapport du L.A.A.S. à Toulouse, $\mathrm{N}^{\circ}$ 87131, mai 1987.

[10] Liou J. J., Lindholm F. A., PARK J. S., IEEE ED34 (1987) 1571-1579.
[11] Leturq P., Rey G., Physique des Composants Actifs à Semiconducteurs (Dunod Université, Paris) 1978.

[12] KuZnicki Z. T., Thin Solid Films 85 (1981) 169-179.

[13] KuZnicki Z. T., Bulletin de l'Académie Polonaise des Sciences; Série des sciences techniques 29, 11-12 (1981) 111-118.

[14] Gunn J. B., J. Electron. Control. 4 (1958) 17-50.

[15] KuZNicki Z. T., Détermination des Régions de Transition et des Profils des Concentrations des Porteurs Libres dans les Structures Multicouches de Silicium ; Rapport de l'Institut de Technologie des Matériaux Electroniques à Varsovie ; 1985 (en Polonais).

[16] KUZNICKI Z. T., en cours de publication.

[17] Kuznicki Z. T., Rev. Phys. Appl. 23 (1988) 13131316.

[18] Stern F., Phys. Rev. B 5 (1972) 4891-4899.

[19] Ando T., Fowler A. B., Stern F., Rev. Mod. Phys. 54 (1982) 2.

[20] ChANDRA A., Solid-State Electron. 23 (1979) 516-517.

[21] Ehlers D. H., Mills D. L., Phys. Rev. B 34 (1986) 3939-3947.

[22] Gummel H. K., IEEE ED-11 (1964) 455-465.

[23] De MARI A., Solid-State Electron 11 (1968) 33-58.

[24] BARNES J., LOMAX R. J., IEEE ED-24 (1977) 10821089. 